UG-21/98

QMW-PH-98-41

SPIN-98/15

December, 1998

\title{
The Kaluza-Klein Monopole in a Massive IIA Background
}

\author{
Eduardo Eyras [ \\ Institute for Theoretical Physics \\ University of Groningen \\ Nijenborgh 4, 9747 AG Groningen, The Netherlands \\ and \\ Yolanda Lozano 2 \\ Spinoza Institute \\ University of Utrecht \\ Leuvenlaan 4, 3508 TD Utrecht, The Netherlands
}

\begin{abstract}
We construct the effective action of the KK-monopole in a massive Type IIA background. We follow two approaches. First we construct a massive M-theory KK-monopole from which the IIA monopole is obtained by double dimensional reduction. This eleven dimensional monopole contains two isometries: one under translations of the TaubNUT coordinate and the other under massive transformations of the embedding coordinates. Secondly, we construct the massive T-duality rules that map the Type IIB NS-5-brane onto the massive Type IIA KK-monopole. This provides a check of the action constructed from eleven dimensions.
\end{abstract}

\footnotetext{
${ }^{1}$ E-mail address: E.A.Eyras@phys.rug.nl

${ }^{2}$ E-mail address: Y.Lozano@phys.uu.nl
} 


\section{Introduction}

The eleven-dimensional interpretation of the massive Type IIA supergravity of Romans [1] has devoted a lot of attention recently. It was shown in [2] that it is not possible to construct a covariant supergravity theory in eleven dimensions with cosmological constant. One might assume that the mass arises in the Type IIA theory from the dimensional reduction procedure, à la Scherk-Schwarz, however a theory was obtained through this kind of construction in [3] with the result that it was not Romans' supergravity but a different massive supergravity for which there is no action.

A massive eleven-dimensional supergravity was proposed in [4] with the peculiarity that it can only be formulated when the eleventh direction is compact, with Lorentz invariance taking place in the other ten dimensional coordinates. This feature circumvents the no-go theorem of [2]. The explicit eleven-dimensional supergravity action depends on the Killing vector associated to translations along the eleventh coordinate, and gives the massive Type IIA supergravity action after a direct dimensional reduction along this direction.

More recently [5] a proposal for massive M-theory has been given based on the connection between M-theory and Type IIB and the relation between the Scherk-Schwarz reduction of Type IIB and the reduction of massive Type IIA. It remains an interesting open problem to make contact with the description of massive eleven-dimensional supergravity with a Killing vector.

M-branes propagating in a massive background were constructed in [6, 4] and referred to generically as massive M-branes. Their effective actions are described by gauged sigma-models in which the Killing isometry is gauged. They contain as well new couplings proportional to the mass which can be interpreted in terms of "massive" solitons.

Furthermore, two massive branes in the Type IIA theory can be obtained from a single massive M-brane [4]. They are obtained as either direct or double dimensional reduction of the massive M-brane effective action along the space-time coordinate where the isometry is realized.

In this paper we will construct the effective action of the massive Type IIA KK-monopole. We will follow two approaches. First of all, we will obtain the action of the IIA KK-monopole by performing a double dimensional reduction in the effective action of the massive eleven-dimensional KK-monopole. As explained in [7] the construction of a massive KK-monopole in eleven dimensions is more subtle than that of an ordinary brane, since the monopole is already described by a gauged sigma-model in the massless case.

The massive M-KK-monopole giving rise to the massive D6-brane after a direct dimensional reduction was constructed in [7]. There it was shown that 
in order to assure invariance under massive gauge transformations the gauge field associated to the Taub-NUT isometry had to transform proportionally to the mass. Also, new couplings to the Born-Infeld field had to be introduced.

In this eleven-dimensional massive monopole the "mass isometry direction" coincides with the Taub-NUT direction, and the dimensionally reduced action gives the massive D6-brane. However, in order to obtain a ten dimensional KK-monopole we are not interested in eliminating the isometry in the Taub-NUT direction once a double dimensional reduction is performed. Therefore we first have to find the worldvolume action of a more general M-KK-monopole in which the invariance under massive transformations is achieved by gauging an isometry other than the Taub-NUT isometry. Double dimensional reduction along this new isometry direction will give rise to the action of the massive IIA KK-monopole (see Figure 1). We present these actions in sections 2, 3 and 1 .

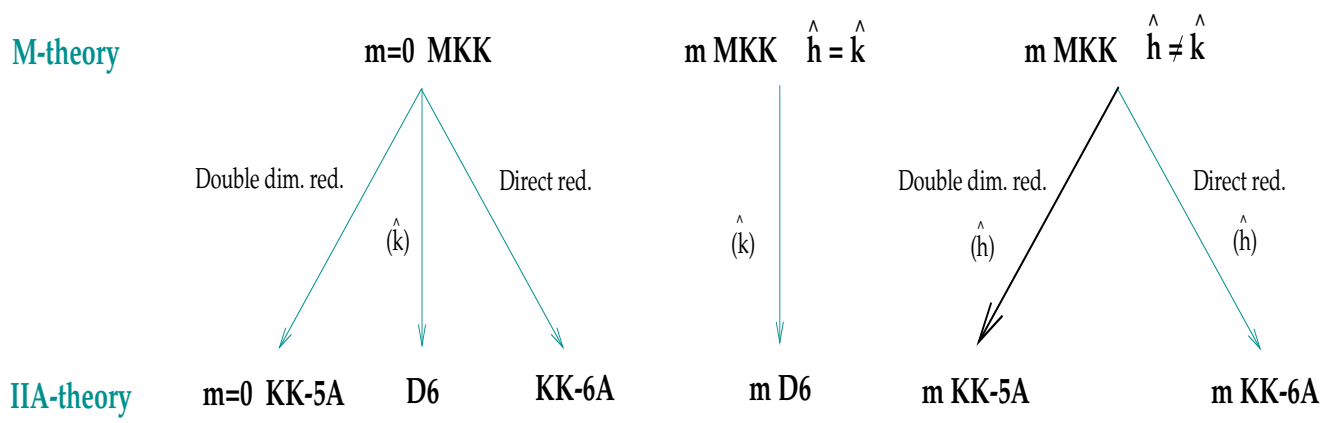

Figure 1: Dimensional reductions of the (massive) M-KK-monopole. In this figure we display the reductions of the massless M-KK-monopole and its two possible massive extensions. The massless M-KK-monopole is described by a gauged sigma-model with Killing vector $\hat{k}$. Reducing along a worldvolume coordinate gives rise to the Type IIA KK-monopole, the reduction along $\hat{k}$ gives the D6-brane and the reduction along a transversal coordinate different than $\hat{k}$ gives a 6-brane (KK-6A) [8, 9, 10, 11], described by a gauged sigma-model. The KK-6A brane is not associated to a central charge in the Type IIA supersymmetry algebra [12]. There are two possible massive extensions depending on whether the massive isometry, $\hat{h}$, is chosen to be $\hat{h}=\hat{k}$ or $\hat{h} \neq \hat{k}$, such that reducing along $\hat{h}$ gives a massive Type IIA brane. When $\hat{h}=\hat{k}$ we obtain a massive D6-brane. When $\hat{h} \neq \hat{k}$ we obtain a massive IIA KK-monopole (KK-5A in the Figure), if $\hat{h}$ lies in a worldvolume direction of the M-KK-monopole; or a massive KK-6A brane, if $\hat{h}$ lies in a transversal direction.

An alternative way to derive the action of the massive IIA KK-monopole is to start with the action of the IIB NS-5-brane constructed in [13] and 
perform a (massive) T-duality transformation. The massive T-duality rules for the target space (dual) potentials that couple to the 5-brane are given in Appendix A. We present the details of the calculation of the massive IIA KK-monopole action by this procedure in section 5. The result coincides with that given in section 4 following the double dimensional reduction approach (see Figure 2).

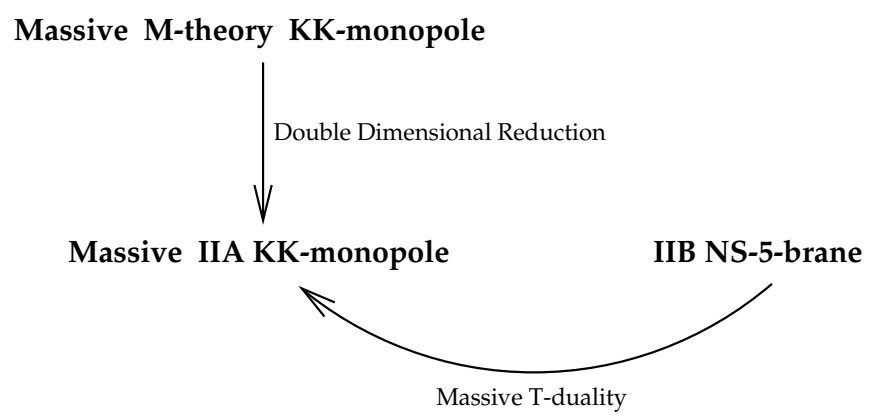

Figure 2: Derivation of the massive IIA KK-monopole. In this Figure we show the two procedures that we have followed to obtain the massive IIA KKmonopole. In the first one we perform a double dimensional reduction along the $\hat{h}$ direction of the massive M-KK-monopole with two gauged isometries, the TaubNUT $\hat{k}$ and $\hat{h}$. In the second case, we obtain the same massive IIA KK-monopole via a massive T-duality transformation on the IIB NS-5-brane effective action.

\section{The Massive M-KK-monopole}

Let us start by recalling the action of the massless M-KK-monopole constructed in [14] [. The KK-monopole in eleven dimensions behaves like a 6-brane, and its field content is that of the 7-dimensional vector multiplet, involving 3 scalars and 1 vector. Since the embedding coordinates describe $11-7=4$ degrees of freedom one scalar has to be eliminated by gauging an isometry of the background

The Taub-NUT space of the monopole is isometric in its Taub-NUT direction. Let us denote $\hat{k}$ the Killing vector associated to this isometry:

$$
\delta \hat{X}^{\hat{\mu}}=-\hat{\sigma}^{(0)} \hat{k}^{\hat{\mu}},
$$

\footnotetext{
${ }^{3}$ For some recent work on KK-monopoles see 15.

${ }^{4}$ For constructions of gauged sigma models with WZ term in arbitrary dimensions see [16.
} 
such that the Lie derivatives of all target space fields and gauge parameters with respect to $\hat{k}$ vanish. The effective action of the monopole is constructed by replacing ordinary derivatives by covariant derivatives:

$$
\partial_{\hat{\imath}} \hat{X}^{\hat{\mu}} \rightarrow D_{\hat{\imath}} \hat{X}^{\hat{\mu}}=\partial_{\hat{\imath}} \hat{X}^{\hat{\mu}}+\hat{A}_{\hat{\imath}} \hat{k}^{\hat{\mu}},
$$

with $\hat{A}_{\hat{\imath}}$ a dependent gauge field given by:

$$
\hat{A}_{\hat{\imath}}=|\hat{k}|^{-2} \partial_{\hat{\imath}} \hat{X}^{\hat{\mu}} \hat{k}_{\hat{\mu}}
$$

where $|\hat{k}|^{2}=-\hat{k}^{\hat{\mu}} \hat{k}^{\hat{\nu}} \hat{g}_{\hat{\mu} \hat{\nu}}$. Accordingly, the gauge transformation of $\hat{A}_{\hat{\imath}}$ is:

$$
\delta \hat{A}_{\hat{\imath}}=\partial_{\hat{\imath}} \hat{\sigma}^{(0)} .
$$

In this way the effective metric becomes:

$$
\hat{g}_{\hat{\mu} \hat{\nu}} D_{\hat{\imath}} \hat{X}^{\hat{\mu}} D_{\hat{\jmath}} \hat{X}^{\hat{\nu}}=\hat{\Pi}_{\hat{\mu} \hat{\nu}} \partial_{\hat{\imath}} \hat{X}^{\hat{\mu}} \partial_{\hat{\jmath}} \hat{X}^{\hat{\nu}}
$$

with

$$
\hat{\Pi}_{\hat{\mu} \hat{\nu}}=\hat{g}_{\hat{\mu} \hat{\nu}}+|\hat{k}|^{-2} \hat{k}_{\hat{\mu}} \hat{k}_{\hat{\nu}} .
$$

Since $\hat{\Pi}_{\hat{\mu} \hat{\nu}} \hat{k}^{\hat{\nu}}=0$ the isometry direction is effectively eliminated from the action.

The construction of the $\hat{\sigma}^{(0)}$-invariant WZ term has been given in [7]. We summarize the target space and worldvolume field content in Tables 1 and 2.

The resulting effective action gives the D6-brane of the Type IIA theory after a direct dimensional reduction along the Taub-NUT direction [14].

In [7] the effective action of the M-KK-monopole giving rise to the D6brane of the massive Type IIA theory was also constructed. As discussed in [4] eleven-dimensional massive branes are described by gauged sigma models with gauge coupling constant proportional to $m$. The massive D6-brane is obtained by reducing the massive KK-monopole in which the two Killing vectors associated to the mass and the Taub-NUT isometries coincide. The gauge field $\hat{A}$ must be attributed mass transformation rules and extra terms need to be added to the WZ part. As we mentioned in the Introduction this massive M-KK-monopole cannot give rise to the massive Type IIA KKmonopole after a double dimensional reduction, since the gauged isometry disappears in the reduction. However it is possible to construct a massive M-KK-monopole in which the two isometry directions associated to the mass and the Taub-NUT space are different. Double dimensionally reducing along the direction associated to the mass will give rise to the effective action of the massive IIA KK-monopole. 


\begin{tabular}{|c|c|}
\hline $\begin{array}{c}\text { Target space } \\
\text { Field }\end{array}$ & $\begin{array}{c}\text { Gauge } \\
\text { Parameter }\end{array}$ \\
\hline$\hat{g}_{\hat{\mu} \hat{\nu}}$ & \\
\hline$\hat{C}_{\hat{\mu} \hat{\nu} \hat{\rho}}$ & $\hat{\chi}_{\hat{\mu} \hat{\nu}}$ \\
\hline$\left(i_{\hat{k}} \hat{C}\right)_{\hat{\mu} \hat{\nu}}$ & $\left(i_{\hat{k}} \hat{\chi}\right)_{\hat{\mu}}$ \\
\hline$\overline{\tilde{C}}_{\hat{\mu}_{1} \ldots \hat{\mu}_{6}}$ & $\hat{\tilde{\chi}}_{\hat{\mu}_{1} \ldots \hat{\mu}_{5}}$ \\
\hline$\left(i_{\hat{k}} \hat{\tilde{C}}\right)_{\hat{\mu}_{1} \ldots \hat{\mu}_{5}}$ & $\left(i_{\hat{k}} \hat{\tilde{\chi}}\right)_{\hat{\mu}_{1} \ldots \hat{\mu}_{4}}$ \\
\hline$\hat{N}_{\hat{\mu}_{1} \ldots \hat{\mu}_{8}}$ & $\hat{\Omega}_{\hat{\mu}_{1} \ldots \hat{\mu}_{7}}$ \\
\hline$\left(i_{\hat{k}} \hat{\tilde{N}}\right)_{\hat{\mu}_{1} \ldots \hat{\mu}_{7}}$ & $\left(i_{\hat{k}} \hat{\Omega}\right)_{\hat{\mu}_{1} \ldots \hat{\mu}_{6}}$ \\
\hline
\end{tabular}

Table 1: Target space fields in the M-KK-monopole. This table shows the 11-dimensional target space fields that couple to the M-KK-monopole, together with their gauge parameters. We also include the contractions with the Killing vector $\hat{k}^{\hat{\mu}}$. The field $\hat{\tilde{C}}$ is the Poincaré dual of $\hat{C}$, the 3 -form of eleven-dimensional supergravity, and $\hat{N}$ is the Poincaré dual of the Killing vector, considered as a 1 -form $\hat{k}_{\hat{\mu}}$.

It was shown in [4] that a massive brane in eleven dimensions is obtained by gauging an isometry generated by a Killing vector $\hat{h}$ :

$$
\delta_{\hat{\rho}^{(0)}} \hat{X}^{\hat{\mu}}=\frac{m}{2} l_{p}^{2} \hat{\rho}^{(0)} \hat{h}^{\hat{\mu}}
$$

through the introduction of a gauge field $\hat{b}_{\hat{\imath}}$ transforming as용

$$
\delta \hat{b}_{\hat{\imath}}=\partial_{\hat{\imath}} \hat{\rho}^{(0)}-l_{p}^{-2}\left(i_{\hat{h}} \hat{\chi}\right)_{\hat{\imath}}
$$

where $\left(i_{\hat{h}} \hat{\chi}\right)$ is (the pull-back of) the interior product of the gauge parameter of the eleven-dimensional 3 -form $\hat{C}$ with the Killing vector $\hat{h}$. Substituting ordinary derivatives by covariant derivatives

$$
\mathcal{D}_{\hat{\imath}} \hat{X}^{\hat{\mu}}=\partial_{\hat{\imath}} \hat{X}^{\hat{\mu}}-\frac{m}{2} l_{p}^{2} \hat{b}_{\hat{\imath}} \hat{h}^{\hat{\mu}}
$$

one assures invariance under massive transformations:

$$
\delta_{\hat{\chi}} \hat{L}_{\hat{\mu}_{1} \ldots \hat{\mu}_{r}}=r \frac{m}{2}(-1)^{r}\left(i_{\hat{h}} \hat{\chi}\right)_{\left[\hat{\mu}_{1}\right.}\left(i_{\hat{h}} \hat{L}\right)_{\left.\hat{\mu}_{2} \ldots \hat{\mu}_{r}\right]},
$$

\footnotetext{
${ }^{5}$ Here $l_{p}$ is the eleven-dimensional Planck length.
} 


\begin{tabular}{|c|c|}
\hline $\begin{array}{c}\text { Worldvolume } \\
\text { Field }\end{array}$ & $\begin{array}{c}\sharp \text { of } \\
\text { d.o.f }\end{array}$ \\
\hline \hline$\hat{X}^{\hat{\mu}}$ & $11-7-(1)=3$ \\
\hline$\hat{\omega}_{\hat{\imath}}^{(1)}$ & $7-2=5$ \\
\hline$\hat{\omega}_{\hat{\imath}_{1} \ldots \hat{\imath}_{6}}^{(6)}$ & - \\
\hline
\end{tabular}

Table 2: Worldvolume fields. In this table we summarize the worldvolume fields, together with their degrees of freedom, that occur in the worldvolume action of the M-theory KK-monopole. The worldvolume scalars $\hat{X}^{\hat{\mu}}$ are the embedding coordinates, $\hat{\omega}^{(1)}$ is a 1 -form and $\hat{\omega}^{(6)}$ is a non propagating 6 -form that describes the tension of the monopole. Due to the gauging the embedding scalars describe 3 and not 4 degrees of freedom as indicated in the table.

for a rank $r 11 \operatorname{dim}$ form $\hat{L}$, and

$$
\delta_{\hat{\chi}} \hat{g}_{\hat{\mu} \hat{\nu}}=-m\left(i_{\hat{h}} \hat{\chi}\right)_{(\hat{\mu}}\left(i_{\hat{h}} \hat{g}\right)_{\hat{\nu})},
$$

for the 11 dim metric.

These transformations give rise to the known massive transformations of the Type IIA background fields after dimensional reduction. Together with the gauging it is necessary to include additional $\hat{b}$-dependent terms (proportional to the mass) to achieve invariance under massive transformations.

This suggests that we should construct the massive M-KK-monopole by substituting:

$$
\hat{\Pi}_{\hat{\mu} \hat{\nu}} \partial_{\hat{\imath}} \hat{X}^{\hat{\mu}} \partial_{\hat{\jmath}} \hat{X}^{\hat{\nu}} \rightarrow \hat{\Pi}_{\hat{\mu} \hat{\nu}} \mathcal{D}_{\hat{\imath}} \hat{X}^{\hat{\mu}} \mathcal{D}_{\hat{\jmath}} \hat{X}^{\hat{\nu}}
$$

with $\mathcal{D} \hat{X}$ as defined above. This can also be written as:

$$
\hat{\Pi}_{\hat{\mu} \hat{\nu}} \mathcal{D}_{\hat{\imath}} \hat{X}^{\hat{\mu}} \mathcal{D}_{\hat{\jmath}} \hat{X}^{\hat{\nu}}=\hat{g}_{\hat{\mu} \hat{\nu}} D_{\hat{\imath}} \hat{X}^{\hat{\mu}} D_{\hat{\jmath}} \hat{X}^{\hat{\nu}}
$$

with

$$
D_{\hat{\imath}} \hat{X}^{\hat{\mu}} \equiv \partial_{\hat{\imath}} \hat{X}^{\hat{\mu}}+\hat{A}_{\hat{\imath}} \hat{k}^{\hat{\mu}}-\frac{m}{2} l_{p}^{2} \hat{b}_{\hat{\imath}} \hat{h}^{\hat{\mu}}
$$

and:

$$
\hat{A}_{\hat{\imath}}=|\hat{k}|^{-2} \hat{k}_{\hat{\mu}}\left(\partial_{\hat{\imath}} \hat{X}^{\hat{\mu}}-\frac{m}{2} l_{p}^{2} \hat{b}_{\hat{\imath}} \hat{h}^{\hat{\mu}}\right) .
$$

$\hat{\Pi}_{\hat{\mu} \hat{\nu}}$ transforms as a metric under massive transformations iff 


$$
\delta \hat{k}_{\hat{\mu}}=-\frac{m}{2}\left(i_{\hat{h}} \hat{\chi}\right)_{\hat{\mu}}\left(i_{\hat{h}} \hat{k}\right),
$$

which implies that the Killing vector associated to the Taub-NUT isometry must be attributed a massive transformation:

$$
\delta \hat{k}^{\hat{\mu}}=\frac{m}{2}\left(i_{\hat{k}} i_{\hat{h}} \hat{\chi}\right) \hat{h}^{\hat{\mu}}
$$

The transformation rule (2.16) is that of a vector under massive transformations (see (2.10)). We showed in [7] that $\hat{k}_{\hat{\mu}}$ had to be considered as a target space 1-form in order to construct the action of the $11 \mathrm{dim}$ KK-monopole. In particular, the monopole is charged with respect to its dual 8-form[? We see here that this is also the case regarding massive transformations. With this transformation rule the interior product of $\hat{k}$ with any eleven-dimensional $r$-form transforms according to (2.10) (see Appendix B).

The fact that $\hat{k}^{\hat{\mu}}$ transforms under massive transformations implies that the Killing condition and the massive transformations do not commute?.

In the system of adapted coordinates to the isometry generated by $\hat{h}$ : $\hat{h}^{\hat{\mu}}=\delta_{y}^{\hat{\mu}}$, we can define:

$$
\hat{k}^{y}=\frac{m}{2} l_{p}^{2} \hat{\omega}^{(0)}
$$

with

$$
\delta \hat{\omega}^{(0)}=\frac{1}{2 \pi \alpha^{\prime}}\left(i_{\hat{k}} i_{\hat{h}} \hat{\chi}\right) .
$$

In the reduction to ten dimensions $\hat{k}^{\mu}$ (with $\mu$ a ten dimensional index) will be the Killing vector generating translations along the Taub-NUT direction, and $\hat{\omega}^{(0)}$ an extra worldvolume scalar needed to compensate for certain massive transformations.

It is easy to check that the invariance under $\hat{\sigma}^{(0)}$ is preserved if the $\hat{b}$ field transforms as:

$$
\delta \hat{b}_{\hat{\imath}}=\partial_{\hat{\imath}} \hat{\rho}^{(0)}-\hat{\sigma}^{(0)} \partial_{\hat{\imath}} \hat{\omega}^{(0)}-l_{p}^{-2}\left(i_{\hat{h}} \hat{\chi}\right)_{\hat{\imath}}
$$

The gauge transformation rule of $\hat{A}_{\hat{\imath}}$ (given by (2.15)) is still $\delta \hat{A}_{\hat{\imath}}=\partial_{\hat{\imath}} \hat{\sigma}^{(0)}$, as in the massless case.

\footnotetext{
${ }^{6}$ To be precise, with respect to its interior product with the Killing vector $\hat{k}$.

${ }^{7}$ They commute if $\left(i_{\hat{k}} i_{\hat{h}} \hat{\chi}\right)=0$, which is not the most general case. Note, however, that if in ten dimensions we set the component of the RR 1-form along the Taub-NUT direction to zero, which can always be done since $C^{(1)}$ is a non-physical Stueckelberg field [1], 17, then this condition is satisfied.

${ }^{8}$ In the kinetic term. We will comment later on the WZ term.
} 
The action that we propose for the massive M-theory KK-monopole is the following:

$$
\begin{aligned}
\hat{S}= & -T_{\mathrm{mMKK}} \int d^{7} \hat{\xi} \hat{k}^{2} \sqrt{\left|\operatorname{det}\left(D_{\hat{\imath}} \hat{X}^{\hat{\mu}} D_{\hat{\jmath}} \hat{X} \hat{\nu} \hat{g}_{\hat{\mu} \hat{\nu}}+l_{p}^{2}|\hat{k}|^{-1} \hat{\mathcal{K}}_{\hat{\imath} \hat{\jmath}}^{(2)}\right)\right|} \\
& +\frac{1}{7 !} l_{p}^{2} T_{\mathrm{mMKK}} \int d^{7} \hat{\xi} \varepsilon^{\hat{\imath}_{1} \ldots \hat{\imath}_{7}} \hat{\mathcal{K}}_{\hat{\imath}_{1} \ldots \hat{\imath}_{7}}^{(7)} .
\end{aligned}
$$

The covariant derivatives are defined by (2.14) and $\hat{\mathcal{K}}^{(2)}$ is the massive field strength of the worldvolume field $\hat{\omega}^{(1)}$ :

$$
\hat{\mathcal{K}}^{(2)}=2 \partial \hat{\omega}^{(1)}+l_{p}^{-2} D \hat{X}^{\hat{\mu}} D \hat{X}^{\hat{\nu}}\left(i_{\hat{k}} \hat{C}\right)_{\hat{\mu} \hat{\nu}}-m l_{p}^{2} \partial \hat{\omega}^{(0)} \hat{b} .
$$

Finally, $\hat{\mathcal{K}}^{(7)}$ is given by:

$$
\begin{aligned}
\hat{\mathcal{K}}^{(7)}= & 7 \partial \hat{\omega}^{(6)}+m \hat{\omega}^{(7)}-\frac{3}{2} l_{p}^{2} m \hat{d}^{(5)}\left(2 \partial \hat{\omega}^{(1)}-m l_{p}^{2} \partial \hat{\omega}^{(0)} \hat{b}\right) \\
& -\frac{1}{7} l_{p}^{-2} D \hat{X}^{\hat{\mu}_{1}} \ldots D \hat{X}^{\hat{\mu}_{7}}\left(i_{\hat{k}} \hat{N}\right)_{\hat{\mu}_{1} \ldots \hat{\mu}_{7}}+3 D \hat{X}^{\hat{\mu}_{1}} \ldots D \hat{X}^{\hat{\mu}_{5}}\left(i_{\hat{k}} \hat{\tilde{C}}\right)_{\hat{\mu}_{1} \ldots \hat{\mu}_{5}} \hat{\mathcal{K}}^{(2)} \\
& -5 l_{p}^{-2} D \hat{X}^{\hat{\mu}_{1}} \ldots D \hat{X}^{\hat{\mu}_{7}} \hat{C}_{\hat{\mu}_{1} \ldots \hat{\mu}_{3}}\left(i_{\hat{k}} \hat{C}\right)_{\hat{\mu}_{4} \hat{\mu}_{5}}\left(i_{\hat{k}} \hat{C}\right)_{\hat{\mu}_{6} \hat{\mu}_{7}} \\
& -15 D \hat{X}^{\hat{\mu}_{1}} \ldots D \hat{X}^{\hat{\mu}_{5}} \hat{C}_{\hat{\mu}_{1} \ldots \hat{\mu}_{3}}\left(i_{\hat{k}} \hat{C}\right)_{\hat{\mu}_{4} \hat{\mu}_{5}}\left(2 \partial \hat{\omega}^{(1)}-m l_{p}^{2} \partial \hat{\omega}^{(0)} \hat{b}\right) \\
& -60 l_{p}^{2} D \hat{X}^{\hat{\mu}_{1}} \ldots D \hat{X}^{\hat{\mu}_{3}} \hat{C}_{\hat{\mu}_{1} \ldots \hat{\mu}_{3}} \partial \hat{\omega}^{(1)}\left(\partial \hat{\omega}^{(1)}-m l_{p}^{2} \partial \hat{\omega}^{(0)} \hat{b}\right) \\
& \left.-60 l_{p}^{4} \hat{A}\left(2 \partial \hat{\omega}^{(1)}-3 m l_{p}^{2} \partial \hat{\omega}^{(0)} \hat{b}\right) \partial \hat{\omega}^{(1)} \partial \hat{\omega}^{(1)}\right\} .
\end{aligned}
$$

This action is invariant under the gauge transformations given in Appendices $\mathrm{B}$ and C.1. Invariance under $\hat{\rho}^{(0)}$ transformations is assured by the presence of covariant derivatives. It can also be seen that with the transformation rule (2.20) for the $\hat{b}$ field the action is invariant under $\hat{\sigma}^{(0)}$ transformations.

Notice that we have introduced a new auxiliary field, $\hat{d}^{(5)}$, associated to the dual massive transformations with parameter $\left(i_{\hat{h}} \hat{\Sigma}\right)$ (see [⿴囗十). This field transforms proportionally to $\left(i_{\hat{k}} i_{\hat{h}} \hat{\Sigma}\right.$ ) (see Appendix C.1), since only contractions with the Taub-NUT Killing vector need to be cancelled in the KK-monopole action.

Moreover, $\hat{b}$ is a non-propagating field in this action, playing the role of gauge field for the $\hat{h}$ isometry?. We will see in the next section that it

${ }^{9}$ Additional $m \hat{b}$ couplings are necessary as well in the WZ term to cancel certain gauge variations. 
disappears in the double dimensionally reduced action. This means that there are no fundamental strings ending on the monopole.

When $\hat{k}$ and $\hat{h}$ are parallel, as in [7], $\hat{\omega}^{(1)}$ transforms like $\hat{b}$ (in (2.20)) प0 and it is not necessary to introduce this new field. Furthermore, an extra term $-15 m l_{p}^{6} \hat{b} \partial \hat{b} \partial \hat{b} \partial \hat{b}$ in the WZ part of the action accounts for the variation of the $\hat{\omega}^{(7)}$ field巴, which is also not needed. This is also the case for $\hat{\omega}^{(0)}$ and $\hat{d}^{(5)}$.

In Table 3 we summarize the worldvolume fields present in (2.21). All these fields can be given an interpretation in terms of solitons on the KKmonopole.

\begin{tabular}{|c|c|}
\hline Worldvolume & Field \\
\hline Field & Strength \\
\hline$\hat{\omega}_{\hat{\imath}}^{(1)}$ & $\overline{\hat{\mathcal{K}}_{\hat{\imath} \hat{\jmath}}^{(2)}}$ \\
\hline$\hat{d}_{\hat{\imath}_{1} \hat{\imath}_{2}}^{(5)}$ & \\
\hline$\hat{\omega}_{\hat{\imath}_{1} \ldots \hat{\nu}_{6}}^{(6)}$ & $\hat{\mathcal{K}}_{\hat{\imath}_{1} \ldots \hat{\imath}_{7}}^{(7)}$ \\
\hline 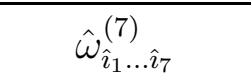 & \\
\hline
\end{tabular}

Table 3: Worldvolume field content of the massive M-theory KKmonopole. In this table we give the worldvolume fields, together with their field strengths, present in the worldvolume action of the massive M-theory KKmonopole.

We find, as in the massless case, a 1 -form $\hat{\omega}^{(1)}$, describing a 0-brane soliton in the worldvolume of the KK-monopole. Its dual 4-form describes a 3-brane soliton. They correspond to the intersections: (0|M2, MKK) and (3|M5, MKK), respectively. The monopole contains as well a 4-brane soliton which couples to the 5 -form dual to one of the embedding scalars: $(4 \mid \mathrm{MKK}, \mathrm{MKK})_{1,2}$. All these intersections have been discussed in [18, 19.

In the massive case the field $\hat{d}^{(5)}$ couples to the 5-brane soliton represented by the configuration 12$]$ [20, 19]:

$$
(5 \mid \mathrm{M} 9, \mathrm{MKK})=\left\{\begin{array}{c|cccccccccc}
\times & \times & \times & \times & \times & z & - & \times & \times & \times & \times \\
\times & \times & \times & \times & \times & \times & \times & z & - & - & -
\end{array}\right.
$$

\footnotetext{
${ }^{10}$ In this case $\hat{\omega}^{(0)}=0$.

${ }^{11}$ With the modification of the transformation law of $\hat{\omega}^{(6)}$ found in $[7$.

${ }^{12}$ We use here a notation where $\times(-)$ indicates a worldvolume (transverse) direction. The first $x$ in a row indicates the time direction.
} 
Here the $z$-direction in the monopole corresponds to the isometry direction of the Taub-NUT space. A single M9-brane contains as well a Killing isometry in its worldvolume, as has been discussed in [21, 22, 23]. This isometric direction has been depicted as well as a $z$-direction. The 5-brane soliton predicted by the M-KK and M9-brane worldvolume supersymmetry algebras [19 is realized as a 4-brane soliton given that it cannot develop a worldvolume direction along the isometry of the M9-brane. This is in agreement with the worldvolume field content that we have found for the massive M-KKmonopole, since the only worldvolume field to which this soliton can couple is the 5 -form $\hat{d}^{(5)}$.

The 6 -form $\hat{\omega}^{(6)}$ is interpreted as the tension of the monopole and couples to the 5-brane soliton realized as the embedding of an M5-brane on the KK-monopole [24, 18]. In the massive case it also plays the role of Stueckelberg field for the auxiliary field $\hat{\omega}^{(7)}$ (see their $\hat{\rho}^{(6)}$ transformation rules in Appendix C.1).

Finally, $\hat{\omega}^{(7)}$ couples to the 6 -brane soliton describing the embedding of the monopole in an M9-brane: $(6 \mid \mathrm{M} 9, \mathrm{MKK})$. The M9-brane contains a 1form vector field in its worldvolume [23]. The dual of this massive 1-form in the nine dimensional worldvolume is a 7 -form field which from the point of view of the M9-brane is the worldvolume field that couples to the 6-brane soliton.

\section{Double Dimensional Reduction: Massive MKK $\rightarrow$ Massive KK-5A}

We can now proceed and perform the double dimensional reduction of the action constructed in the previous section. We will see that the Killing isometry associated to translations of the Taub-NUT coordinate is restored in this process.

In adapted coordinates $\hat{h}^{\hat{\mu}}=\delta_{y}^{\hat{\mu}}$, we take the ansatz for the worldvolume reduction:

$$
\hat{X}^{y}=Y=\hat{\xi}^{6}
$$

with all other worldvolume fields and gauge parameters independent of $\hat{\xi}^{6}$.

The $\hat{k}$ transformation rule (2.17) implies that this vector gets a $y$-component under massive gauge transformations. Therefore we reduce it as:

$$
\hat{k}^{\mu}=k^{\mu}, \quad \hat{k}^{y}=m 2 \pi \alpha^{\prime} \omega^{(0)} .
$$

$k^{\mu}$ will be the Killing vector associated to the isometry of the Taub-NUT space of the IIA KK-monopole. 
In order to keep track of all the gauge transformations we have to introduce a compensating gauge transformation:

$$
\delta \hat{\xi}^{\hat{\imath}}=\delta^{\hat{\imath} 6}\left[-\Lambda^{(0)}+\frac{m}{2}\left(2 \pi \alpha^{\prime}\right) \rho^{(0)}-\frac{m}{2}\left(2 \pi \alpha^{\prime}\right) \sigma^{(0)} \omega^{(0)}\right],
$$

where $\Lambda^{(0)}$ is a g.c.t. in the direction $Y$.

The reduction rules for the background fields and gauge parameters can be found for instance in [7]. The worldvolume fields reduce as:

$$
\begin{array}{rlrl}
l_{p}^{2} \hat{b}_{i} & =2 \pi \alpha^{\prime} b_{i}, & l_{p}^{2} \hat{b}_{6} & =2 \pi \alpha^{\prime} v^{(0)}, \\
l_{p}^{2} \hat{\omega}_{i}^{(1)} & =2 \pi \alpha^{\prime} \omega_{i}^{(1)}, & l_{p}^{2} \hat{\omega}_{6}^{(1)}=2 \pi \alpha^{\prime} \omega^{(0)}, \\
l_{p}^{2} \hat{d}_{i_{1} \ldots i_{5}}^{(5)}=2 \pi \alpha^{\prime} d_{i_{1} \ldots i_{5}}^{(5)}, & l_{p}^{2} \hat{\omega}_{i_{1} \ldots i_{5} 6}^{(6)}=2 \pi \alpha^{\prime} \omega_{i_{1} \ldots i_{5}}^{(5)} \\
l_{p}^{2} \hat{\omega}_{i_{1} \ldots i_{6} 6}^{(7)}=-\frac{3}{7}\left(2 \pi \alpha^{\prime}\right)^{2} v^{(0)} \partial_{\left[i_{1}\right.} \omega_{\left.i_{2} \ldots i_{6}\right]}^{(5)}+\frac{6}{7}\left(2 \pi \alpha^{\prime}\right)\left(1-\frac{m}{2}\left(2 \pi \alpha^{\prime}\right) v^{(0)}\right) \omega_{i_{1} \ldots i_{6}}^{(6)} .
\end{array}
$$

The (modified) gauge transformations of the new, reduced, worldvolume fields can be found in Appendix C.2.

The reduction of $\hat{\omega}^{(6)}$ holds up to a total derivative (see Appendix C). The reduction of $\hat{d}_{i_{1} \ldots i_{4} 6}^{(5)}$ gives a worldvolume 4 -form which is gauge invariant and contributes to the reduced action with a decoupled term, therefore we have fixed it to zero. The scalar field $v^{(0)}$ also has vanishing transformation law. However this field contributes to the double dimensionally reduced action as:

$$
\int d^{6} \xi\left(1-\frac{m}{2}\left(2 \pi \alpha^{\prime}\right) v^{(0)}\right) \mathcal{L}_{\mathrm{mAKK}}
$$

and is constrained to a constant by the equation of motion of the worldvolume field $\omega^{(5)}$ playing the role of tension of the IIA monopole. In general it modifies the tension of the new massive p-brane as [4]:

$$
T_{\text {mIIA }}=\left(1-\frac{m}{2}\left(2 \pi \alpha^{\prime}\right) v^{(0)}\right) T_{\mathrm{mM}} .
$$

This has the implication that for the particular value $v^{(0)}=2 / m\left(2 \pi \alpha^{\prime}\right)$ the brane tension vanishes. The physical mechanism behind this phenomenon is unclear and it would be interesting to investigate (see the Conclusions for a further discussion).

The dependent gauge field $\hat{A}$ reduces as: 


$$
\begin{aligned}
\hat{A}_{i}= & \left(1+e^{2 \phi}|k|^{-2}\left(i_{k} C^{(1)}+m \pi \alpha^{\prime} \omega^{(0)}\right)^{2}\right)^{-1} \times \\
& \times\left(A_{i}-e^{2 \phi}|k|^{-2}\left(C_{i}^{(1)}-m \pi \alpha^{\prime} b_{i}\right)\left(i_{k} C^{(1)}+m \pi \alpha^{\prime} \omega^{(0)}\right)\right),
\end{aligned}
$$

where $A_{i} \equiv|k|^{-2} k_{\mu} \partial_{i} X^{\mu}$, and

$$
\begin{aligned}
\hat{A}_{6}= & -\left(1+e^{2 \phi}|k|^{-2}\left(i_{k} C^{(1)}+m \pi \alpha^{\prime} \omega^{(0)}\right)^{2}\right)^{-1} \times \\
& \times e^{2 \phi}|k|^{-2}\left(1-m \pi \alpha^{\prime} v^{(0)}\right)\left(i_{k} C^{(1)}+m \pi \alpha^{\prime} \omega^{(0)}\right) .
\end{aligned}
$$

\section{The Action of the Massive IIA KK-monopole}

The double dimensional reduction of the action of the massive M-KK-monopole gives:

$$
\begin{gathered}
S=-T_{\mathrm{mAKK}} \int d^{6} \xi k^{2} e^{-2 \phi} \sqrt{1+e^{2 \phi} k^{-2}\left(i_{k} C^{(1)}+m \pi \alpha^{\prime} \omega^{(0)}\right)^{2}} \times \\
\times \sqrt{\left|\operatorname{det}\left(\Pi_{i j}-\left(2 \pi \alpha^{\prime}\right)^{2} k^{-2} \mathcal{K}_{i}^{(1)} \mathcal{K}_{j}^{(1)}+\frac{\left(2 \pi \alpha^{\prime}\right) k^{-1} e^{\phi}}{\sqrt{1+e^{2 \phi} k^{-2}\left(i_{k} C^{(1)}+m \pi \alpha^{\prime} \omega^{(0)}\right)^{2}}} \mathcal{K}_{i j}^{(2)}\right)\right|} \\
\quad+\frac{1}{6 !}\left(2 \pi \alpha^{\prime}\right) T_{\mathrm{mAKK}} \int d^{6} \xi \epsilon^{i_{1} \ldots i_{6}} \mathcal{K}_{i_{1} \ldots i_{6}}^{(6)} .
\end{gathered}
$$

The covariant derivative is defined as: $D_{i} X^{\mu}=\partial_{i} X^{\mu}+A_{i} k^{\mu}$ and

$$
\Pi_{i j}=D_{i} X^{\mu} D_{j} X^{\nu} g_{\mu \nu} .
$$

The tension $T_{\mathrm{mAKK}}$ is the modified tension given by (3.6).

The gauge invariant forms $\mathcal{K}^{(2)}$ and $\mathcal{K}^{(1)}$ are the field strengths of $\omega^{(1)}$ and $\omega^{(0)}$, respectively:

$$
\begin{aligned}
\mathcal{K}^{(2)}= & 2 \partial \omega^{(1)}+\frac{1}{2 \pi \alpha^{\prime}}\left(i_{k} C^{(3)}\right)-2 \mathcal{K}^{(1)}\left(D X C^{(1)}\right) \\
& +\frac{m}{2} \omega^{(0)}(D X D X B)-m\left(2 \pi \alpha^{\prime}\right) \omega^{(0)} \partial \omega^{(0)} A, \\
\mathcal{K}^{(1)}= & \partial \omega^{(0)}-\frac{1}{2 \pi \alpha^{\prime}}\left(i_{k} B\right),
\end{aligned}
$$

and the WZ term is the field strength of the worldvolume field $\omega^{(5)}$, playing the role of tension of the IIA KK-monopole: 


$$
\begin{aligned}
\mathcal{K}^{(6)}= & 6\left(\partial \omega^{(5)}+m \omega^{(6)}\right)-3 m\left(2 \pi \alpha^{\prime}\right) d^{(5)} \partial \omega^{(0)}+\frac{1}{2 \pi \alpha^{\prime}}\left(i_{k} N\right) \\
& -15\left(i_{k} C^{(5)}\right)\left(2 \partial \omega^{(1)}+\frac{1}{2 \pi \alpha^{\prime}}\left(i_{k} C^{(3)}\right)+\frac{m}{2} \omega^{(0)} B\right) \\
& -6\left(\left(i_{k} \tilde{B}\right)-\frac{m}{2}\left(2 \pi \alpha^{\prime}\right) \omega^{(0)} C^{(5)}\right) \mathcal{K}^{(1)} \\
& -60\left(2 \pi \alpha^{\prime}\right) D X^{\mu} D X^{\nu} D X^{\rho} C_{\mu \nu \rho}^{(3)} \mathcal{K}^{(1)} \mathcal{K}^{(2)} \\
& +\frac{30}{2 \pi \alpha^{\prime}} B\left(i_{k} C^{(3)}\right)^{2}+30 D X^{\mu} D X^{\nu} D X^{\rho} C_{\mu \nu \rho}^{(3)}\left(i_{k} C^{(3)}\right) \mathcal{K}^{(1)} \\
& -180\left(2 \pi \alpha^{\prime}\right) D X^{\mu} D X^{\nu} B_{\mu \nu}\left(\partial \omega^{(1)}\right)^{2} \\
& +\frac{20}{2 \pi \alpha^{\prime}} C^{(3)}\left(i_{k} B\right)\left(i_{k} C^{(3)}+m \pi \alpha^{\prime} \omega^{(0)} B\right) \\
& -\frac{15}{4} m^{2}\left(2 \pi \alpha^{\prime}\right)\left(\omega^{(0)}\right)^{2} D X^{\mu_{1}} \ldots D X^{\mu_{6}} B_{\mu_{1} \ldots \mu_{6}}^{3} \\
& -45 m\left(2 \pi \alpha^{\prime}\right) \omega^{(0)} D X^{\mu_{1}} \ldots D X^{\mu_{4}} B_{\mu_{1} \ldots \mu_{4}}^{2} \partial \omega^{(1)} \\
& +\frac{15}{2} m \omega^{(0)} B^{2}\left(i_{k} C^{(3)}\right)-360\left(2 \pi \alpha^{\prime}\right)^{2} A\left(\partial \omega^{(1)}+\frac{m}{4} \omega^{(0)} B\right)^{2} \partial \omega^{(0)} \\
& +15\left(2 \pi \alpha^{\prime}\right)^{2} \frac{e^{2 \phi}|k|^{-2}\left(i_{k} C^{(1)}+m \pi \alpha^{\prime} \omega^{(0)}\right)}{1+e^{2 \phi}|k|^{-2}\left(i_{k} C^{(1)}+m \pi \alpha^{\prime} \omega^{(0)}\right)^{2}} \mathcal{K}^{(2)} \mathcal{K}^{(2)} \mathcal{K}^{(2)}
\end{aligned}
$$

In Table 4 we summarize the worldvolume field content. The gauge transformation rules of background and worldvolume fields can be found in Appendices B, C.2 and reference [4]. We summarize our notation for the Type IIA background fields in Table 5 .

The action of the ten dimensional IIA KK-monopole is manifestly invariant under translations of the Taub-NUT coordinate, since all the reduced fields and gauge parameters have vanishing Lie derivative with respect to $k$. This symmetry has been restored in the reduction by a mechanism in which the $y$ component of the Killing vector in eleven dimensions gives rise to an auxiliary worldvolume field that is needed to compensate the massive variation of the Stueckelberg field $i_{k} C^{(1)}$. A further check of this action is that for $m=0$ reduces to the action of the massless IIA KK-monopole [7].

It is also worth noting that the field $b$ has disappeared in the reduced action. This reflects the fact that there are no fundamental strings ending on the monopole. Nevertheless there is a string-like object, described by $\omega^{(1)}$, ending on the monopole. In fact, the worldvolume fields that couple to the 


\begin{tabular}{|c|c|}
\hline $\begin{array}{c}\text { Worldvolume } \\
\text { Field }\end{array}$ & $\begin{array}{c}\text { Field } \\
\text { Strength }\end{array}$ \\
\hline \hline$\omega^{(0)}$ & $\mathcal{K}_{i}^{(1)}$ \\
\hline$\omega_{i}^{(1)}$ & $\mathcal{K}_{i j}^{(2)}$ \\
\hline$d^{(5)}$ & \\
\hline$\omega_{i_{1} \ldots i_{5}}^{(5)}$ & $\mathcal{K}_{i_{1} \ldots i_{6}}^{(6)}$ \\
\hline$\omega_{i_{1} \ldots i_{6}}^{(6)}$ & \\
\hline
\end{tabular}

Table 4: Worldvolume fields of the massive IIA KK-monopole. In this table we give the worldvolume fields, together with their field strengths, that occur in the effective action of the massive IIA KK-monopole.

\begin{tabular}{|c|c|c|c|}
\hline $\begin{array}{c}\text { Target space } \\
\text { Field }\end{array}$ & $\begin{array}{c}\text { Gauge } \\
\text { Parameter }\end{array}$ & $\begin{array}{c}\text { Dual } \\
\text { Field }\end{array}$ & $\begin{array}{c}\text { Gauge } \\
\text { Parameter }\end{array}$ \\
\hline \hline$g_{\mu \nu}, \phi$ & - & - & - \\
\hline$B_{\mu \nu}$ & $\Lambda_{\mu}$ & $\tilde{B}_{\mu_{1} \ldots \mu_{6}}$ & $\tilde{\Lambda}_{\mu_{1} \ldots \mu_{5}}$ \\
\hline$C_{\mu}^{(1)}$ & $\Lambda^{(0)}$ & $C_{\mu_{1} \ldots \mu_{7}}^{(7)}$ & $\Lambda_{\mu_{1} \ldots \mu_{6}}^{(6)}$ \\
\hline$C_{\mu \nu \rho}^{(3)}$ & $\Lambda_{\mu \nu}^{(2)}$ & $C_{\mu_{1} \ldots \mu_{5}}^{(5)}$ & $\Lambda_{\mu_{1} \ldots \mu_{4}}^{(4)}$ \\
\hline$k_{\mu}$ & - & $N_{\mu_{1} \ldots \mu_{7}}$ & $\Omega_{\mu_{1} \ldots \mu_{6}}^{(6)}$ \\
\hline
\end{tabular}

Table 5: Target space fields of the type IIA superstring. The type IIA background contains the NS-NS sector: $\left(g_{\mu \nu}, \phi, B_{\mu \nu}\right)$, the RR sector: $\left(C^{(1)}, C^{(3)}\right)$, and the Poincaré duals of the RR fields and the NS-NS 2-form $B:\left(C^{(5)}, C^{(7)}, \tilde{B}\right)$. The Kaluza-Klein monopole couples to a new field $N$, dual to the Killing vector associated to the Taub-NUT isometry, considered as a 1-form $k_{\mu}$.

soliton solutions of a KK-monopole are those necessary to construct invariant field strengths for the fields $i_{k} C^{(p+1)}$ (see [13]). These field strengths have the form:

$$
\mathcal{K}^{(p)}=p \partial \omega^{(p-1)}+\frac{1}{2 \pi \alpha^{\prime}}\left(i_{k} C^{(p+1)}\right)+\ldots
$$


so that $\omega^{(p-1)}$ couples to a $(p-2)$-brane soliton which describes the boundary of a $p$-brane ending on the monopole, with one of its worldvolume directions wrapped around the Taub-NUT direction of the monopole. Since the target space field associated to $\omega^{(1)}$ is $\left(i_{k} C^{(3)}\right)$ it describes a wrapped D2-brane ending on the monopole.

We also find the soliton configurations: (2|D4, KK), (3|NS5, KK) and $(3 \mid \mathrm{KK}, \mathrm{KK})_{1,2}$ (see [25, 13]). The only modifications due to the mass occur in the explicit expressions of the field strengths, where new terms proportional to the mass appear, which involve worldvolume fields that already propagated in the massless case. There is however an exception, and that is the presence of the $d^{(5)}$ worldvolume field associated to the dual massive transformations, which couples to a 4-brane soliton. This soliton is a domain wall in the six dimensional worldvolume and is described by the configuration:

$$
(4 \mid \mathrm{D} 8, \mathrm{KK})=\left\{\begin{array}{c|cccccccccc}
\times & \times & \times & \times & \times & - & \times & \times & \times & \times \\
\times & \times & \times & \times & \times & \times & z & - & - & -
\end{array}\right.
$$

which is obtained by reducing the $(4 \mid \mathrm{M} 9, \mathrm{KK})$ soliton configuration of the M-theory KK-monopole along the isometric direction of the M9-brane. This intersection is related to the Hanany-Witten configuration [26]:

$$
\begin{array}{ll|l}
\text { D5 : } & \times & \times \times---\times \times \times- \\
\text { NS5: } & \times & \times \times \times \times \times---- \\
\text { D3: } & \times & \times \times------\times
\end{array}
$$

by T-duality along the 3,4,9 directions. It is also T-dual to the intersection (4|D7, NS5) in Type IIB, corresponding to a 4-brane soliton in the IIB NS5 -brane [27.

There is as well another 4-brane soliton in the KK-monopole worldvolume [18 which is already present in the massless case. This is the embedding of the D4-brane on the monopole: $(4 \mid \mathrm{D} 4, \mathrm{KK})$, and it couples to the worldvolume field $\omega^{(5)}$, describing the tension of the monopole. This is the reduction of the 5-brane soliton $(5 \mid \mathrm{M} 5, \mathrm{KK})$, which couples to the tension $\hat{\omega}^{(6)}$ of the M-KK-monopole. Finally, the reduction of the 6-brane soliton (6|M9, KK) gives a 5-brane soliton realized as the embedding of the KK-monopole on a KK-7A-brane[3.

\footnotetext{
${ }^{13}$ This brane is obtained by reducing the M9-brane along a worldvolume direction other than the $z$-direction, but it is not predicted by the Type IIA supersymmetry algebra. As discussed in [23], this is also the case for the KK-6A brane, obtained by reducing the M-KK-monopole along a transverse direction different from the Taub-NUT direction. These branes are required by U-duality of M-theory on a d-torus, as it has been shown in [8, 9, 10].
} 


\section{Massive $T$-duality: IIB NS-5 $\rightarrow$ Massive IIAKK}

In this section we obtain the massive IIA KK-monopole through a "massive" T-duality transformation in the action of the IIB NS-5-brane.

The action of the Type IIB NS-5-brane was constructed in [13] and is given by:

$$
\begin{aligned}
S= & -T_{\mathrm{NS}-5 \mathrm{~B}} \int d^{6} \xi e^{-2 \varphi} \sqrt{1+e^{2 \varphi}\left(C^{(0)}\right)^{2}} \times \\
& \times \sqrt{\left|\operatorname{det}\left(\mathrm{g}-\left(2 \pi \alpha^{\prime}\right) \frac{e^{\varphi}}{\sqrt{1+e^{2 \varphi}\left(C^{(0)}\right)^{2}}} \tilde{\mathcal{F}}\right)\right|} \\
& +\frac{1}{6 !}\left(2 \pi \alpha^{\prime}\right) T_{\mathrm{NS}-5 \mathrm{~B}} \int d^{6} \xi \epsilon^{i_{1} \ldots i_{6}} \tilde{\mathcal{G}}_{i_{1} \ldots i_{6}}^{(6)} .
\end{aligned}
$$

Here $\tilde{\mathcal{F}}=2 \partial c^{(1)}+\frac{1}{2 \pi \alpha^{\prime}} C^{(2)}$ and:

$$
\begin{aligned}
\tilde{\mathcal{G}}^{(6)}= & \left\{6 \partial \tilde{c}^{(5)}-\frac{1}{2 \pi \alpha^{\prime}} \tilde{\mathcal{B}}-\frac{45}{2\left(2 \pi \alpha^{\prime}\right)} \mathcal{B} C^{(2)} C^{(2)}-15 C^{(4)} \tilde{\mathcal{F}}\right. \\
& -180\left(2 \pi \alpha^{\prime}\right) \mathcal{B} \partial c^{(1)} \partial c^{(1)}-90 \mathcal{B} C^{(2)} \partial c^{(1)} \\
& \left.+15\left(2 \pi \alpha^{\prime}\right)^{2} \frac{C^{(0)}}{e^{-2 \varphi}+\left(C^{(0)}\right)^{2}} \tilde{\mathcal{F}} \tilde{\mathcal{F}} \tilde{\mathcal{F}}\right\} .
\end{aligned}
$$

In Table 6 we have summarized our notation for the Type IIB background fields.

\begin{tabular}{|c|c|c|c|}
\hline $\begin{array}{c}\text { Target space } \\
\text { Field }\end{array}$ & $\begin{array}{c}\text { Gauge } \\
\text { Parameter }\end{array}$ & $\begin{array}{c}\text { Dual } \\
\text { Field }\end{array}$ & $\begin{array}{c}\text { Gauge } \\
\text { Parameter }\end{array}$ \\
\hline \hline $\mathrm{g}_{\mu \nu}, \varphi$ & - & - & - \\
\hline $\mathcal{B}_{\mu \nu}$ & $\Lambda_{\mu}$ & $\tilde{\mathcal{B}}_{\mu_{1} \ldots \mu_{6}}$ & $\tilde{\Lambda}_{\mu_{1} \ldots \mu_{5}}$ \\
\hline$C^{(0)}$ & - & - & - \\
\hline$C_{\mu \nu}^{(2)}$ & $\Lambda_{\mu}^{(1)}$ & $C_{\mu_{1} \ldots \mu_{6}}^{(6)}$ & $\Lambda_{\mu_{1} \ldots \mu_{5}}^{(5)}$ \\
\hline
\end{tabular}

Table 6: Target space fields of the type IIB superstring. The Type IIB background contains the common sector: $\left(\mathrm{g}_{\mu \nu}, \varphi, \mathcal{B}_{\mu \nu}\right)$, the RR sector: $\left(C^{(0)}, C^{(2)}, C^{(4)}\right)$, and the Poincaré duals of the 2 -forms $C^{(2)}$ and $\mathcal{B}:\left(C^{(6)}, \tilde{\mathcal{B}}\right)$. 
We apply now a T-duality transformation along a transverse direction. The worldvolume fields do not change rank after T-duality since the original and dual branes have the same number of worldvolume dimensions. Moreover, we have a new scalar field $Z^{\prime}$, which is the T-dual of the coordinate along which we perform the duality transformation. The T-duality rules for the worldvolume fields are given by:

$$
\begin{aligned}
Z^{\prime}= & \left(2 \pi \alpha^{\prime}\right) \omega^{(0)} \\
c^{(1)^{\prime}=} & -\omega^{(1)}-\frac{m}{4}\left(2 \pi \alpha^{\prime}\right)\left(\omega^{(0)}\right)^{2} \partial Z \\
\partial \tilde{c}^{(5) \prime}= & \partial \omega^{(5)}+60\left(2 \pi \alpha^{\prime}\right)^{2} \partial Z \partial \omega^{(1)} \partial \omega^{(1)} \partial \omega^{(0)} \\
& +m \omega^{(6)}-\frac{1}{2} m\left(2 \pi \alpha^{\prime}\right) d^{(5)} \partial \omega^{(0)} .
\end{aligned}
$$

Here $Z$ is the Taub-NUT coordinate of the KK-monopole. Its occurrence on the right hand side of the two expressions above is required by gauge invariance, and assures the gauged sigma-model structure necessary to describe the KK-monopole.

Using the massive T-duality rules given in (A.1) and (A.3) for the background fields, we find the following transformations for the worldvolume curvatures:

$$
\tilde{\mathcal{F}}^{\prime}=-\mathcal{K}^{(2)}, \quad \tilde{\mathcal{G}}^{(6) \prime}=\mathcal{K}^{(6)},
$$

where $\mathcal{K}^{(2)}$ is given by $(4.3)$ and $\mathcal{K}^{(6)}$ by (4.4). Substituting in the IIB NS-5brane effective action we recover the expression (4.1) for the effective action of the massive IIA KK-monopole that we obtained from eleven dimensions. This provides a check of that action as well as of the massive T-duality rule (A.3) given in the Appendix.

One remark is in order at this point. In general the double dimensional reduction of a massive M-brane gives a tension [4]:

$$
T=\left(1-\frac{m}{2}\left(2 \pi \alpha^{\prime}\right) v^{(0)}\right) \hat{T} \int d \hat{\xi}
$$

for the reduced brane, where $\hat{\xi}$ is the compact worldvolume direction. A particular example is the massive IIA KK-monopole obtained in the previous section. This suggests that in the massive case the tensions should transform under T-duality as:

$$
T_{\mathrm{B} 5}^{\prime}=T_{\mathrm{mAKK}}=\left(1-\frac{m}{2}\left(2 \pi \alpha^{\prime}\right) v^{(0)}\right) T_{\mathrm{mMKK}},
$$


which seems, however, an arbitrary choice from the T-duality point of view. We leave some especulations about this point for the Conclusions.

\section{Conclusions}

We have constructed the worldvolume effective action of the Type IIA KKmonopole propagating in a background with non-vanishing cosmological constant. The worldvolume field content of this brane consists on a scalar, a 1 -form, two 5-forms and a 6-form. One of the two 5-forms is interpreted as the tension of the monopole, whereas the other one is associated to dual massive transformations in the worldvolume of the monopole. The 6-form and the tension of the monopole are interpreted as a Stueckelberg pair with respect to massive transformations.

These worldvolume fields have been interpreted in terms of soliton solutions propagating in the worldvolume of the monopole. The new feature with respect to the massless case is that there is a 4-brane soliton realized as the domain wall intersection of the KK-monopole with a D8-brane, and a 6-brane soliton corresponding to the embedding of the monopole on a KK7-brane.

We have already mentioned that the KK7-brane belongs to a particular class of Type IIA brane solutions which are not predicted by the spacetime supersymmetry algebra. It is unclear why this happens. These branes have been encountered already in the literature in a different context, namely they are required in order to fill up multiplets of BPS states in representations of the U-duality symmetry group of M-theory on a d-torus [8, 9, 10, 11]. As we discuss in [23], where we calculate the kinetic part of their worldvolume effective actions, these branes do not have an obvious interpretation in weakly coupled string theory since they scale with the coupling constant more singularly than $1 / g_{s}^{2}$.

The derivation of the KK-monopole effective action from eleven dimensions gives a tension depending on the mass and on a Wilson line of the gauge field associated to the massive isometry (which is set to a constant value by the integration of the worldvolume 5 -form playing the role of tension of the monopole) (see (3.6)). In particular, the tension can vanish for a certain value of the Wilson line. In fact, this is a general feature for the Type IIA massive branes that are obtained from eleven dimensions by a double dimensional reduction [4]. The presence of covariant derivatives with respect to massive transformations in the eleven-dimensional massive branes implies that in the double dimensional reduction the brane can be wrapped along the eleventh direction with unusual winding number, which depends on the mass and on a Wilson line of the gauge field. Therefore, consistency implies 
that $\left(1-\frac{m}{2}\left(2 \pi \alpha^{\prime}\right) v^{(0)}\right)$ has to be an integer, i.e. the winding number, with the tension of the reduced brane proportional to it. The Wilson line may be interpreted as a distance $2 \pi \alpha^{\prime} v^{(0)}$ corresponding to a D8-brane, since a massive IIA brane is a brane in the presence of D8-branes. This is similar to the interpretation of the masses of the string states in terms of the D8-brane positions in a Type I' construction. Since the D8-branes are T-dual to the D9-branes, the arbitrariness of $v^{(0)}$ from the T-duality point of view might be originated by Wilson lines in Type IIB with D9-branes.

Finally it would be of interest to analyze the six dimensional interacting gauge theory that arises from considering a system of parallel massive KKmonopoles in the limit in which gravity is decoupled [28].

\section{Acknowledgements}

We would like to thank E. Bergshoeff for useful discussions. The work of E.E. is part of the research program of the Dutch Foundation FOM.

\section{A T-duality}

In this Appendix we summarize the massive T-duality rules for the background fields given in [17, 29, 30] [4. We also derive the massive T-duality transformation of the $\mathcal{B}$ field coupled to the IIB NS-5-brane. In our notation $z$ is the direction along which we perform the duality transformation.

The following T-duality rules from Type IIB $[$ onto massive Type IIA backgrounds have already been constructed in the literature:

\footnotetext{
${ }^{14}$ The T-duality rules between Type IIA and Type IIB backgrounds were derived in 31 for the massless case.

${ }^{15} \mathrm{In}$ the basis in which $C^{(4)}$ is S-duality invariant.
} 


$$
\begin{aligned}
& C^{(0) \prime}=-C_{z}^{(1)}-\frac{m}{2} \tilde{Z}, \\
& C_{\mu z}^{(2) \prime}=-C_{\mu}^{(1)}+\left(C_{z}^{(1)}+\frac{m}{2} \tilde{Z}\right) \frac{g_{\mu z}}{g_{z z}}, \quad e^{\varphi^{\prime}}=\frac{1}{\sqrt{\left|g_{z z}\right|}} e^{\phi}, \\
& C_{\mu \nu}^{(2) \prime}=-C_{\mu \nu z}^{(3)}+2 C_{[\mu}^{(1)} B_{\nu] z} \quad \mathcal{B}_{\mu \nu}^{\prime}=B_{\mu \nu}-\frac{2}{g_{z z}} B_{[\mu z} g_{\nu] z}, \\
& -2 C_{z}^{(1)} \frac{g_{z[\mu}}{g_{z z}} B_{\nu] z} \quad \mathcal{B}_{\mu z}^{\prime}=-\frac{g_{\mu z}}{g_{z z}}, \\
& -\frac{m}{2} \tilde{Z}\left(B_{\mu \nu}-2 B_{[\mu z} \frac{g_{\nu] z}}{g_{z z}}\right), \quad \mathrm{g}_{\mu \nu}^{\prime}=g_{\mu \nu}-\frac{1}{g_{z z}}\left(g_{\mu z} g_{\nu z}-B_{\mu z} B_{\nu z}\right), \\
& C_{\mu \nu \rho z}^{(4) \prime}=-C_{\mu \nu \rho}^{(3)}+\frac{3}{2} C_{[\mu \nu z}^{(3)} \frac{g_{\rho] z}}{g_{z z}} \quad \mathrm{~g}_{\mu z}^{\prime}=-\frac{1}{g_{z z}} B_{\mu z}, \\
& +\frac{3}{2}\left(C_{[\mu}^{(1)}-C_{z}^{(1)} \frac{g_{[\mu z}}{g_{z z}}\right) B_{\nu \rho]}, \\
& \mathrm{g}_{z z}^{\prime}=\frac{1}{g_{z z}} \\
& C_{\mu_{1} \ldots \mu_{4}}^{(4) \prime}=-C_{\mu_{1} \ldots \mu_{4} z}^{(5)}+4\left(C_{\left[\mu_{1} \mu_{2} \mu_{3}\right.}^{(3)}-3 C_{\left[\mu_{1} \mu_{2} z\right.}^{(3)} \frac{g_{\mu_{3} z}}{g_{z z}}\right) B_{\left.\mu_{4}\right] z} \\
& +3 C_{\left[\mu_{1} \mu_{2} z\right.}^{(3)}\left(B_{\left.\mu_{3} \mu_{4}\right]}-2 B_{\mu_{3} z} \frac{g_{\left.\mu_{4}\right] z}}{g_{z z}}\right)-6\left(C_{\left[\mu_{1}\right.}^{(1)}-C_{z}^{(1)} \frac{g_{\left[\mu_{1} z\right.}}{g_{z z}}\right) B_{\mu_{2} \mu_{3}} B_{\left.\mu_{4}\right] z}, \\
& C_{\mu_{1} \ldots \mu_{5} z}^{(6) \prime}=-C_{\mu_{1} \ldots \mu_{5}}^{(5)}+5 C_{\left[\mu_{1} \ldots \mu_{4} z\right.}^{(5)} \frac{g_{\left.\mu_{5}\right] z}}{g_{z z}} \\
& -15 C_{\left[\mu_{1} \mu_{2} z\right.}^{(3)} B_{\mu_{3} \mu_{4}} \frac{g_{\left.\mu_{5}\right] z}}{g_{z z}} \\
& +\frac{15}{2}\left(C_{\left[\mu_{1}\right.}^{(1)}-C_{z}^{(1)} \frac{g_{\left[\mu_{1} z\right.}}{g_{z z}}-\frac{m}{2} \tilde{Z} \frac{g_{\left[\mu_{1} z\right.}}{g_{z z}}\right) B_{\mu_{2} \mu_{3}} B_{\left.\mu_{4} \mu_{5}\right]} .
\end{aligned}
$$

The inverse rules (from massive Type IIA to Type IIB) are given by: 


$$
\begin{aligned}
& C_{z}^{(1) \prime}=-C^{(0)}-\frac{m}{2} \tilde{Z}, \\
& C_{\mu}^{(1) \prime}=-C_{\mu z}^{(2)}+C^{(0)} \mathcal{B}_{\mu z}, \\
& e^{\phi^{\prime}}=\frac{1}{\sqrt{\left|\mathrm{g}_{z z}\right|}} e^{\varphi}, \\
& C_{\mu \nu z}^{(3) \prime}=-C_{\mu \nu}^{(2)}+2 C_{[\mu z}^{(2)} \frac{\mathrm{g}_{\nu] z}}{\mathrm{~g}_{z z}} \\
& g_{\mu \nu}^{\prime}=\mathrm{g}_{\mu \nu}-\frac{1}{\mathrm{~g}_{z z}}\left(\mathrm{~g}_{\mu z} \mathrm{~g}_{\nu z}-\mathcal{B}_{\mu z} \mathcal{B}_{\nu z}\right), \\
& -\frac{m}{2} \tilde{Z}\left(\mathcal{B}_{\mu \nu}-2 \mathcal{B}_{[\mu z} \frac{\mathrm{g}_{\nu] z}}{\mathrm{~g}_{z z}}\right), \quad g_{\mu z}^{\prime}=-\frac{1}{\mathrm{~g}_{z z}} \mathcal{B}_{\mu z}, \\
& C_{\mu \nu \rho}^{(3) \prime}=-C_{\mu \nu \rho z}^{(4)}+\frac{3}{2} C_{[\mu \nu}^{(2)} \mathcal{B}_{\rho] z} \\
& g_{z z}^{\prime}=\frac{1}{\mathrm{~g}_{z z}}, \\
& -\frac{3}{2} \mathcal{B}_{[\mu \nu} C_{\rho] z}^{(2)} \\
& B_{\mu \nu}^{\prime}=\mathcal{B}_{\mu \nu}-\frac{2}{\mathrm{~g}_{z z}} \mathcal{B}_{[\mu z} \mathrm{g}_{\nu] z} \\
& +6 C_{[\mu z}^{(2)} \mathcal{B}_{\nu z} \frac{\mathrm{g}_{\rho] z}}{\mathrm{~g}_{z z}} \\
& B_{\mu z}^{\prime}=-\frac{\mathrm{g}_{z \mu}}{\mathrm{g}_{z z}}, \\
& C_{\mu_{1} \ldots \mu_{4} z}^{(5) \prime}=-C_{\mu_{1} \ldots \mu_{4}}^{(4)}+4 C_{\left[\mu_{1} \mu_{2} \mu_{3} z\right.}^{(4)} \frac{\mathrm{g}_{\left.\mu_{4}\right] z}}{\mathrm{~g}_{z z}}-3 C_{\left[\mu_{1} \mu_{2}\right.}^{(2)} \mathcal{B}_{\left.\mu_{3} \mu_{4}\right]} \\
& -6 C_{z\left[\mu_{1}\right.}^{(2)} \mathcal{B}_{\mu_{2} \mu_{3}} \frac{\mathrm{g}_{\left.\mu_{4}\right] z}}{\mathrm{~g}_{z z}}-6 \mathcal{B}_{z\left[\mu_{1}\right.} C_{\mu_{2} \mu_{3}}^{(2)} \frac{\mathrm{g}_{\left.\mu_{4}\right] z}}{\mathrm{~g}_{z z}} \\
& -\frac{3}{2} m \tilde{Z} \mathcal{B}_{\left[\mu_{1} \mu_{2}\right.} \mathcal{B}_{\left.\mu_{3} \mu_{4}\right]}+6 m \tilde{Z} \mathcal{B}_{\left[\mu_{1} \mu_{2}\right.} \mathcal{B}_{\mu_{3} z} \frac{\mathrm{g}_{\left.\mu_{4}\right] z}}{\mathrm{~g}_{z z}} \\
& C_{\mu_{1} \ldots \mu_{5}}^{(5) \prime}=-C_{\mu_{1} \ldots \mu_{5} z}^{(6)}+5\left(C_{\left[\mu_{1} \ldots \mu_{4}\right.}^{(4)}-4 C_{\left[\mu_{1} \ldots \mu_{3} z\right.}^{(4)} \frac{\mathrm{g}_{\mu_{4} z}}{\mathrm{~g}_{z z}}\right) \mathcal{B}_{\left.\mu_{5}\right] z} \\
& -\frac{15}{2} \mathcal{B}_{\left[\mu_{1} \mu_{2}\right.} \mathcal{B}_{\left.\mu_{3} \mu_{4}\right]} C_{\left.\mu_{5}\right] z}^{(2)}-30 C_{z\left[\mu_{1}\right.}^{(2)} \mathcal{B}_{\mu_{2} \mu_{3}} \mathcal{B}_{\mu_{4} z} \frac{\mathrm{g}_{\left.\mu_{5}\right] z}}{\mathrm{~g}_{z z}}
\end{aligned}
$$

The IIB NS-5-brane couples minimally to the field $\tilde{\mathcal{B}}$, dual to the NS-NS 2-form. Its massive T-duality rules are given by: 


$$
\begin{aligned}
& \tilde{\mathcal{B}}_{\mu_{1} \ldots \mu_{5} z}^{\prime}=\tilde{B}_{\mu_{1} \ldots \mu_{5} z}-5\left(C_{\left[\mu_{1} \ldots \mu_{4} z\right.}^{(5)}-3 B_{\left[\mu_{1} \mu_{2}\right.} C_{\mu_{3} \mu_{4} z}^{(3)}\right)\left(C_{\left.\mu_{5}\right]}^{(1)}-C_{z}^{(1)} \frac{g_{\left.\mu_{5}\right] z}}{g_{z z}}\right) \\
& -5\left(C_{\left[\mu_{1} \mu_{2} \mu_{3}\right.}^{(3)}-\frac{3}{2} C_{\left[\mu_{1} \mu_{2} z\right.}^{(3)} \frac{g_{\left.\mu_{3}\right] z}}{g_{z z}}\right) C_{\mu_{4} \mu_{5} z}^{(3)} \\
& -\frac{m}{2} \tilde{Z}\left(C_{\mu_{1} \ldots \mu_{5}}^{(5)}-5 C_{\left[\mu_{1} \ldots \mu_{4} z\right.}^{(5)} \frac{g_{\left.\mu_{5}\right] z}}{g_{z z}}\right) \\
& +\frac{15}{8} m^{2} \tilde{Z}^{2} B_{\left[\mu_{1} \mu_{2}\right.} B_{\mu_{3} \mu_{4}} \frac{g_{\left.\mu_{5}\right] z}}{g_{z z}}, \\
& \tilde{\mathcal{B}}_{\mu_{1} \ldots \mu_{6}}^{\prime}=-N_{\mu_{1} \ldots \mu_{6} z}-6 \tilde{B}_{\left[\mu_{1} \ldots \mu_{5} z\right.} B_{\left.\mu_{6}\right] z} \\
& +30\left(C_{\left[\mu_{1} \ldots \mu_{4} z\right.}^{(5)}-3 C_{\left[\mu_{1} \mu_{2} z\right.}^{(3)} B_{\mu_{3} \mu_{4}}\right)\left(C_{\mu_{5}}^{(1)}-C_{z}^{(1)} \frac{g_{\mu_{5} z}}{g_{z z}}\right) B_{\left.\mu_{6}\right] z} \\
& +10\left(C_{\left[\mu_{1} \ldots \mu_{3}\right.}^{(3)}-\frac{3}{2} \frac{g_{\left[\mu_{1} z\right.}}{g_{z z}} C_{\mu_{2} \mu_{3} z}^{(3)}\right)\left(C_{\mu_{4} \mu_{5} z}^{(3)} B_{\left.\mu_{6}\right] z}-m \tilde{Z} B_{\mu_{4} \mu_{5}} B_{\left.\mu_{6}\right] z}\right) \\
& +3 m \tilde{Z}\left(C_{\left[\mu_{1} \ldots \mu_{5}\right.}^{(5)}-5 C_{\mu_{1} \ldots \mu_{4} z}^{(5)} \frac{g_{\mu_{5} z}}{g_{z z}}\right) B_{\left.\mu_{6}\right] z} \\
& -30 C_{\left[\mu_{1} \mu_{2} z\right.}^{(3)}\left(C_{\mu_{3} \mu_{4} z}^{(3)}+\frac{m}{2} \tilde{Z} B_{\mu_{3} \mu_{4}}\right) \frac{g_{\mu_{5} z}}{g_{z z}} B_{\left.\mu_{6}\right] z} \\
& -\frac{15}{2}\left(C_{\left[\mu_{1} \mu_{2} z\right.}^{(3)}+\frac{m}{2} \tilde{Z} B_{\left[\mu_{1} \mu_{2}\right.}\right)\left(C_{\mu_{3} \mu_{4} z}^{(3)}+\frac{m}{2} \tilde{Z} B_{\mu_{3} \mu_{4}}\right) B_{\mu_{5} \mu_{6}} \\
& +\frac{45}{4} m^{2} \tilde{Z}^{2} B_{\left[\mu_{1} \mu_{2}\right.} B_{\mu_{3} \mu_{4}} B_{\mu_{5}} \frac{g_{\left.\mu_{6}\right] z}}{g_{z z}} .
\end{aligned}
$$

Let us clarify the role played by $\tilde{Z}$ in the expressions above. In the $T$ duality from the IIB (IIA) NS-5-brane to the IIA (IIB) KK-monopole, $\tilde{Z}$ is the T-dual transformed of the coordinate transversal to the NS-5-brane:

$$
\tilde{Z}=Z^{\prime}=\left(2 \pi \alpha^{\prime}\right) \omega^{(0)}
$$

and not another embedding coordinate. However, in the T-duality from the IIA (IIB) KK-monopole to the IIB (IIA) NS-5-brane, $\tilde{Z}$ is indeed the transversal coordinate to the 5-brane. When the T-duality takes place between two D-branes $\tilde{Z}$ is proportional to the $\sigma$ component of the Born-Infeld field of the dual brane when the reduction is direct, or is equal to $\sigma$ when the reduction is double $\square$.

\footnotetext{
${ }^{16}$ The double dimensional reduction of a Type IIB brane must be a Scherk-Schwarz type of reduction in order to make contact with massive Type IIA.
} 


\section{B Target Space Gauge Symmetries}

The gauge symmetries of the eleven-dimensional background fields that couple to the M-KK-monopole are given by:

$$
\begin{aligned}
\delta \hat{g}_{\hat{\mu} \hat{\nu}}= & -m\left(i_{\hat{h}} \hat{\chi}\right)_{(\hat{\mu}}\left(i_{\hat{h}} \hat{g}\right)_{\hat{\nu})}, \\
\delta \hat{C}= & 3 \partial \hat{\chi}-\frac{3}{2} m\left(i_{\hat{h}} \hat{\chi}\right)\left(i_{\hat{h}} \hat{C}\right) \\
\delta\left(i_{\hat{k}} \hat{C}\right)= & 2 \partial\left(i_{\hat{k}} \hat{\chi}\right)-m\left(i_{\hat{h}} \hat{\chi}\right)\left(i_{\hat{k}} i_{\hat{h}} \hat{C}\right)-m\left(2 \pi \alpha^{\prime}\right) \partial \hat{\omega}^{(0)}\left(i_{\hat{h}} \hat{\chi}\right) \\
\delta\left(i_{\hat{k}} \hat{\tilde{C}}\right)= & 5 \partial\left(i_{\hat{k}} \hat{\tilde{\chi}}\right)+15 \partial \hat{\chi}\left(i_{\hat{k}} \hat{C}\right)-10 \hat{C} \partial\left(i_{\hat{k}} \hat{\chi}\right) \\
& +\frac{5}{2} m\left(i_{\hat{h}} \hat{\chi}\right)\left(i_{\hat{k}} i_{\hat{h}} \hat{\tilde{C}}\right)+\frac{m}{2}\left(i_{\hat{h}} i_{\hat{k}} \hat{\Sigma}\right)-\frac{5}{2} m\left(2 \pi \alpha^{\prime}\right) \partial \hat{\omega}^{(0)}\left(i_{\hat{h}} \hat{\tilde{\chi}}\right) \\
\delta\left(i_{\hat{k}} \hat{N}\right)= & 7\left\{\partial\left(i_{\hat{k}} \hat{\Omega}\right)+15 \partial\left(i_{\hat{k}} \hat{\tilde{\chi}}\right)\left(i_{\hat{k}} \hat{C}\right)+30 \partial \hat{\chi}\left(i_{\hat{k}} \hat{C}\right)\left(i_{\hat{k}} \hat{C}\right)\right. \\
& -20 \hat{C}\left(i_{\hat{k}} \hat{C}\right) \partial\left(i_{\hat{k}} \hat{\chi}\right)+\frac{m}{2}\left(i_{\hat{h}} \hat{\chi}\right)\left(i_{\hat{k}} i_{\hat{h}} \hat{N}\right)-\frac{m}{2}\left(2 \pi \alpha^{\prime}\right) \partial \hat{\omega}^{(0)}\left(i_{\hat{h}} \hat{\Sigma}\right) \\
& +\frac{3}{2} m\left(i_{\hat{h}} i_{\hat{k}} \hat{\Sigma}\right)\left(i_{\hat{k}} \hat{C}\right) \\
& \left.-\frac{15}{2} m\left(2 \pi \alpha^{\prime}\right) \partial \hat{\omega}^{(0)}\left(i_{\hat{h}} \hat{\tilde{\chi}}\right)\left(i_{\hat{k}} \hat{C}\right)+10 m\left(2 \pi \alpha^{\prime}\right) \hat{C}\left(i_{\hat{k}} \hat{C}\right) \partial \hat{\omega}^{(0)}\left(i_{\hat{h}} \hat{\chi} \hat{B}\right\} .2\right)
\end{aligned}
$$

These fields transform as well under $\hat{\rho}^{(0)}$ and $\hat{\sigma}^{(0)}$ transformations in the usual way.

The transformations of the ten dimensional fields coupled to the massive IIA KK-monopole can be found in [4], as well as the relations between tenand eleven- dimensional fields and gauge parameters. The transformation of $i_{k} N$ is however new, and it is given by: 


$$
\begin{aligned}
\delta\left(i_{k} N\right)= & 6 \partial\left(i_{k} \Omega^{(6)}\right)-3 m\left(i_{k} \Sigma^{(6)}\right)\left(i_{k} B\right) \\
& +60 \partial\left(i_{k} \Lambda^{(4)}\right)\left(i_{k} C^{(3)}+\frac{m}{2}\left(2 \pi \alpha^{\prime}\right) \omega^{(0)} B\right) \\
& -30\left(\partial\left(i_{k} \tilde{\Lambda}\right)-\frac{m}{2}\left(2 \pi \alpha^{\prime}\right) \omega^{(0)} \partial \Lambda^{(4)}\right)\left(i_{k} B\right) \\
& -60 \partial \Lambda\left(i_{k} C^{(3)}+\frac{m}{2}\left(2 \pi \alpha^{\prime}\right) \omega^{(0)} B\right)^{2} \\
& +120 \partial \Lambda^{(2)}\left(i_{k} C^{(3)}+\frac{m}{2}\left(2 \pi \alpha^{\prime}\right) \omega^{(0)} B\right)\left(i_{k} B\right) \\
& +60\left(\partial\left(i_{k} \Lambda^{(2)}\right)+\frac{m}{2}\left(2 \pi \alpha^{\prime}\right) \omega^{(0)} \partial \Lambda\right) B\left(i_{k} C^{(3)}+\frac{m}{2}\left(2 \pi \alpha^{\prime}\right) \omega^{(0)} B\right) \\
& +20 \partial\left(i_{k} \Lambda\right) C^{(3)}\left(i_{k} C^{(3)}+\frac{m}{2}\left(2 \pi \alpha^{\prime}\right) \omega^{(0)} B\right) \\
& -40\left(\partial\left(i_{k} \Lambda^{(2)}\right)+\frac{m}{2}\left(2 \pi \alpha^{\prime}\right) \omega^{(0)} \partial \Lambda\right) C^{(3)}\left(i_{k} B\right)
\end{aligned}
$$

where:

$$
\begin{aligned}
\left(i_{\hat{k}} \hat{\Omega}\right)_{\mu_{1} \ldots \mu_{5} y} & =-\left(i_{k} \Omega^{(6)}\right)_{\mu_{1} \ldots \mu_{5}} \\
\left(i_{\hat{k}} \hat{\Sigma}\right)_{\mu_{1} \ldots \mu_{5} y} & =-\left(i_{k} \Sigma^{(6)}\right)_{\mu_{1} \ldots \mu_{5}} .
\end{aligned}
$$

\section{Worldvolume Gauge Symmetries}

Here we give the gauge transformations of the worldvolume fields present in the effective actions of the massive M-theory and Type IIA KK-monopoles. 


\section{C.1 Massive M-KK-monopole}

$$
\begin{aligned}
\delta \hat{\omega}^{(1)}= & \partial \hat{\mu}-\frac{1}{2 \pi \alpha^{\prime}}\left(i_{\hat{k}} \hat{\chi}\right)-\frac{m}{2}\left(2 \pi \alpha^{\prime}\right) \partial \hat{\omega}^{(0)} \hat{\rho}^{(0)}, \\
\delta \hat{d}^{(5)}= & \frac{1}{2 \pi \alpha^{\prime}}\left(i_{\hat{h}} i_{\hat{k}} \hat{\Sigma}\right), \\
\delta \hat{\omega}^{(6)}= & 6 \partial \hat{\rho}^{(5)}-m \hat{\rho}^{(6)}+\frac{1}{2 \pi \alpha^{\prime}}\left(i_{\hat{k}} \hat{\Omega}\right)-30 \hat{\omega}^{(1)} \partial\left(i_{\hat{k}} \hat{\tilde{\chi}}\right) \\
& -180\left(2 \pi \alpha^{\prime}\right) \partial \hat{\chi} \hat{\omega}^{(1)} \partial \hat{\omega}^{(1)}-120\left(2 \pi \alpha^{\prime}\right)^{2} \partial \hat{\sigma}^{(0)} \hat{\omega}^{(1)} \partial \hat{\omega}^{(1)} \partial \hat{\omega}^{(1)}, \\
\delta \hat{\omega}^{(7)}= & \partial \hat{\rho}^{(6)}+15\left(2 \pi \alpha^{\prime}\right)\left(i_{\hat{h}} \hat{\tilde{\chi}}\right) \partial \hat{\omega}^{(0)} \partial \hat{\omega}^{(1)}-\frac{1}{2} \partial \hat{\omega}^{(0)}\left(i_{\hat{h}} \hat{\Omega}\right) \\
& -\frac{3}{2}\left(2 \pi \alpha^{\prime}\right) \hat{d}^{(5)}\left(\frac{2}{2 \pi \alpha^{\prime}} \partial\left(i_{\hat{k}} \hat{\chi}\right)+m \partial\left(i_{\hat{k}} i_{\hat{h}} \hat{\chi}\right) \hat{b}-m \partial \hat{\omega}^{(0)}\left(i_{\hat{h}} \hat{\chi}\right)\right) \\
& -3 \hat{b}\left[\partial\left(i_{\hat{h}} i_{\hat{k}} \hat{\Omega}\right)-5\left(2 \pi \alpha^{\prime}\right) \partial\left(i_{\hat{k}} \hat{\tilde{\chi}}\right) \partial \hat{\omega}^{(0)}-20\left(2 \pi \alpha^{\prime}\right) \partial\left(i_{\hat{h}} i_{\hat{k}} \hat{\tilde{\chi}}\right) \partial \hat{\omega}^{(1)}\right. \\
& +60\left(2 \pi \alpha^{\prime}\right)^{3} \partial \hat{\sigma}^{(0)}\left(\partial \hat{\omega}^{(1)}\right)^{2} \partial \hat{\omega}^{(0)}+60\left(2 \pi \alpha^{\prime}\right)^{2} \partial \hat{\chi} \partial \hat{\omega}^{(1)} \partial \hat{\omega}^{(0)} \\
& \left.+60\left(2 \pi \alpha^{\prime}\right)^{2} \partial\left(i_{\hat{h}} \hat{\chi}\right)\left(\partial \hat{\omega}^{(1)}\right)^{2}\right] .
\end{aligned}
$$

\section{C.2 Massive IIA KK-monopole}

$$
\begin{aligned}
\delta v^{(0)}= & 0, \\
\delta \omega^{(0)}= & \frac{1}{2 \pi \alpha^{\prime}}\left(i_{k} \Lambda\right), \\
\delta \omega^{(1)}= & \partial \mu^{(0)}-\frac{1}{2 \pi \alpha^{\prime}}\left(i_{k} \Lambda^{(2)}\right)-\frac{m}{2} \omega^{(0)} \Lambda+\omega^{(0)}\left(\partial \Lambda^{(0)}+\frac{m}{2}\left(2 \pi \alpha^{\prime}\right) \partial\left(\sigma^{(0)} \omega^{(0)}\right)\right), \\
\delta d^{(5)}= & -\frac{1}{2 \pi \alpha^{\prime}}\left(i_{k} \Sigma^{(6)}\right), \\
\delta \omega^{(5)}= & 5 \partial \rho^{(4)}-m \rho^{(5)}-\frac{1}{2 \pi \alpha^{\prime}}\left(i_{k} \Omega^{(6)}\right)-5 \partial\left(i_{k} \tilde{\Lambda}\right) \omega^{(0)}+20 \omega^{(1)} \partial\left(i_{k} \Lambda^{(4)}\right) \\
& -60\left(2 \pi \alpha^{\prime}\right) \partial \Lambda^{(2)} \omega^{(1)} \partial \omega^{(0)}+60\left(2 \pi \alpha^{\prime}\right) \omega^{(1)} \partial \omega^{(1)} \partial \Lambda \\
& +60\left(2 \pi \alpha^{\prime}\right)^{2} \sigma^{(0)} \partial \omega^{(1)} \partial \omega^{(1)} \partial \omega^{(0)}+\frac{5}{4} m\left(2 \pi \alpha^{\prime}\right)\left(\omega^{(0)}\right)^{2} \partial \Lambda^{(4)}, \\
\delta \omega^{(6)}= & \partial \rho^{(5)}+\frac{1}{2} d^{(5)} \partial\left(i_{k} \Lambda\right) .
\end{aligned}
$$


Finally, the new gauge parameters in (C.1) and (C.2) are related to the eleven-dimensional ones as follows:

$$
\hat{\rho}_{i_{1} \ldots i_{4} 6}^{(5)}=\rho_{i_{1} \ldots i_{4}}^{(4)}, \quad \hat{\rho}_{i_{1} \ldots i_{5} 6}^{(6)}=\rho_{i_{1} \ldots i_{5}}^{(5)} .
$$

\section{References}

[1] L.J. Romans, Massive N=2a Supergravity in Ten Dimensions, Phys. Lett. B169 (1986) 374.

[2] K. Bautier, S. Deser, M. Henneaux and D. Seminara, No cosmological $D=11$ supergravity, Phys. Lett. B406 (1997) 49, hep-th/9704131.

[3] P.S. Howe, N.D. Lambert and P.C. West, A new massive Type IIA supergravity from compactification, Phys. Lett. B416 (1998) 303, hep-th/9707139;

I.V. Lavrinenko, H. Lü and C.N. Pope, Fiber bundles and generalized dimensional reductions, hep-th/9710243.

[4] E. Bergshoeff, Y. Lozano and T. Ortín, Massive Branes, Nucl. Phys. B518 (1998) 363, hep-th/9712115.

[5] C.M. Hull, Massive string theories from M-theory and F-theory, hep-th/9811021.

[6] Y. Lozano, Eleven dimensions from the massive D-2-brane, Phys. Lett. B414 (1997) 52, hep-th/9707011;

T. Ortín, A note on the D-2-brane of the massive type IIA theory and gauged sigma models, Phys. Lett. B415 (1997) 39, hep-th/9707113.

[7] E. Bergshoeff, E. Eyras and Y. Lozano, The Massive Kaluza-Klein Monopole, Phys. Lett. B430 (1998) 7r, hep-th/9802199.

[8] C.M. Hull, U-duality and BPS spectrum of super Yang-Mills theory and M-theory, JHEP 07 (1998) 018, hep-th/9712075.

[9] S. Elitzur, A. Giveon, D. Kutasov and E. Rabinovici, Algebraic aspects of matrix theory on $T^{d}$, Nucl. Phys. B509 (1998) 122, hep-th/9707217; M. Blau and M. O'Loughlin, Aspects of U-duality in matrix theory, Nucl. Phys. B525 (1998) 182, hep-th/9712047; 
N.A. Obers, B. Pioline and E. Rabinovici, M-theory and U-duality on $T^{d}$ with gauge backgrounds, Nucl. Phys. B525 (1998) 163, hep-th/9712084.

[10] N.A. Obers and B. Pioline, U-duality and M-theory, hep-th/9809039.

[11] P. Meessen and T. Ortín, An SL(2,Z) multiplet of nine-dimensional type II supergravity theories, hep-th/9806120.

[12] C.M. Hull, Gravitational Duality, Branes and Charges, Nucl.Phys. B509 (1998) 216, hep-th/9705162.

[13] E. Eyras, B. Janssen and Y. Lozano, 5-branes, KK-monopoles and Tduality, Nucl. Phys. B531 (1998) 275, hep-th/9806169.

[14] E. Bergshoeff, B. Janssen and T. Ortín, Kaluza-Klein Monopoles and Gauged Sigma-Models, Phys. Lett. B410 (1997) 132, hep-th/9706117.

[15] Y. Imamura, Born-Infeld action and Chern-Simons term from Kaluza-Klein monopole in M-theory, Phys. Lett. B414 (1997) 242, hep-th/9706144;

A. Sen, Dynamics of multiple KK-monopoles in $M$ - and string theory, Adv. Theor. Math. Phys. 1 (1998) 115, hep-th/9707042;

A. Hanany and G. Lifschytz, M(atrix) theory on $T^{6}$ and a $m$ (atrix) theory description of KK-monopoles, hep-th/9708037;

I. Brunner and A. Karch, Matrix description of M-theory on $T^{6}$, Phys. Lett. B416 (1998) 67, hep-th/9707259;

R. Gregory, J.A. Harvey and G. Moore, Unwinding strings and T-duality of $K K$ and $H$ monopoles, hep-th/9708086.

[16] C.M. Hull and B.S. Spence, The geometry of the gauged sigma model with Wess-Zumino term, Nucl. Phys. B353 (1991) 379.

[17] E. Bergshoeff, M. de Roo, M. B. Green, G. Papadopoulos and P. K. Townsend, Duality of type II 7-Branes and 8-Branes, Nucl. Phys. B470 (1996) 113, hep-th/9601150.

[18] E. Bergshoeff, M. de Roo, E. Eyras, B. Janssen and J.P. van der Schaar, Intersections involving waves and monopoles in eleven dimensions, Class. Quant. Grav. 14 (1997) 2757, hep-th/9704120. 
[19] E. Bergshoeff, J. Gomis and P.K. Townsend, M-brane intersections from worldvolume superalgebras, Phys. Lett. B421 (1998) 109, hep-th/9711043.

[20] M. de Roo, Intersecting branes and supersymmetry, hep-th/9703124.

[21] E. Bergshoeff and J.P. van der Schaar, On M-9-branes, hep-th/9806069.

[22] E. Bergshoeff, E. Eyras, R. Halbersma, C.M. Hull, Y. Lozano and J.P. van der Schaar, Spacetime-filling branes and strings with sixteen supercharges, in preparation.

[23] E. Eyras and Y. Lozano, Brane actions and string dualities, Contribution to the proceedings of the TMR-meeting Quantum Aspects of Gauge Theories, Supersymmetry and Unification, Corfu, September 1998.

[24] A.A. Tseytlin, Harmonic superpositions of M-branes, Nucl. Phys. B475 (1996) 149, hep-th/9604035.

[25] G. Papadopoulos, T-duality and the worldvolume solitons of five-branes and KK-monopoles, Phys. Lett. B434 (1998) 277, hep-th/9712162.

[26] A. Hanany and E. Witten, Type IIB superstrings, BPS monopoles and three-dimensional gauge dynamics, Nucl. Phys. B492 (1997) 152, hep-th/9611230.

[27] E. Bergshoeff, G. Papadopoulos and J.P. van der Schaar, Domain-walls on the brane, Phys. Lett. B430 (1998) 63, hep-th/9801158.

[28] N. Seiberg, A matrix description of $M$-theory on $T^{5}$ and $T^{5} / Z_{2}$, Phys. Lett. B408 (1997) 98, hep-th/9705221;

E. Witten, New "gauge" theories in six dimensions, JHEP 01 (1998) 001, hep-th/9710065.

[29] E. Bergshoeff and M. de Roo, D-branes and T-duality, Phys. Lett. B380 (1996) 265, hep-th/9603123.

[30] M.B. Green, C.M. Hull and P.K. Townsend, D-p-brane Wess-Zumino actions, T-duality and the cosmological constant, Phys. Lett. B382 (1996) 65 , hep-th/9604119.

[31] E. Bergshoeff, C.M. Hull and T. Ortín, Duality in the type II superstring effective action, Nucl. Phys. B451 (1995) 547, hep-th/9504081. 